Research Paper

\title{
Extravascular compared to Intravascular Femoral Closure is Associated with Less Bleeding and Similar MACE after Percutaneous Coronary Intervention
}

\author{
Seung-Hyun Kim ${ }^{1 *}$, Michael Behnes ${ }^{* \otimes}$, Sebastian Baron ${ }^{1}$, Tetyana Shchetynska-Marinova ${ }^{1}$, Melike Uensal ${ }^{1}$, \\ Kambis Mashayekhi², Ursula Hoffmann ${ }^{1}$, Martin Borggrefe ${ }^{1}$, Ibrahim Akin ${ }^{1}$ \\ 1. First Department of Medicine, University Medical Centre Mannheim (UMM), Faculty of Medicine Mannheim, University of Heidelberg, European Center \\ for AngioScience (ECAS), and DZHK (German Center for Cardiovascular Research) partner site Heidelberg/Mannheim, Mannheim, Germany. \\ 2. Division of Cardiology and Angiology II, University Heart Center Freiburg - Bad Krozingen, Bad Krozingen, Germany. \\ *S.-H. K. and M.Be. contributed equally to this study
}

$\square$ Corresponding author: Michael Behnes MD, First Department of Medicine, University Medical Centre Mannheim (UMM), Faculty of Medicine Mannheim, University of Heidelberg, Theodor-Kutzer-Ufer 1-3, 68167 Mannheim, Germany, Phone: +49 621383 6239, E-mail: michael.behnes@umm.de

(c) Ivyspring International Publisher. This is an open access article distributed under the terms of the Creative Commons Attribution (CC BY-NC) license (https://creativecommons.org/licenses/by-nc/4.0/). See http://ivyspring.com/terms for full terms and conditions.

Received: 2018.08.15; Accepted: 2018.10.13; Published: 2019.01.01

\begin{abstract}
Background: Various types of vascular closure devices (VCDs) are frequently utilized in patients undergoing percutaneous coronary intervention $(\mathrm{PCl})$ in order to prevent arterial access site bleeding, which represents one of the most relevant complications associated with adverse clinical outcomes. This study aims to compare directly two mechanistically different types of femoral closure $(\mathrm{FC})$ devices in patients undergoing $\mathrm{PCl}$.

Methods: This single-center, prospective, observational study includes consecutively patients either treated by the extravascular StarClose SE $®$ (Abbott, Illinois, U.S.A.) or the intravascular AngioSeal ${ }^{\mathrm{TM}}$ FC (St. Jude Medical, Inc., St. Paul, MN, U.S.A.) after PCl. The primary endpoint was bleeding complications, the secondary endpoint was major adverse cardiac events (MACE) at 30 days of follow-up.

Results: 200 patients in each group (StarClose $\mathrm{SE} 囚$ and AngioSeal ${ }^{\mathrm{TM}}$ ) were enrolled following $\mathrm{PCl}$. The rates of overall and non-access site bleedings were significantly higher in the AngioSeal ${ }^{\mathrm{TM}}$ group $(56 \% ; 6 \%)$ compared to the StarClose SE® group (43.5\%; $0.5 \%)(p=0.012 ; 0.003)$. Additionally, complicated access site bleedings were also significantly higher in the AngioSeal ${ }^{\mathrm{TM}}$ group $(p=0.011)$. No significant differences of MACE were observed in both groups. However, there was a higher rate of unsuccessful implantation of the StarClose $S E \circledast(n=12$, excluded from the study).

Conclusions: In case of successful implantation, FC by the AngioSeal ${ }^{\mathrm{TM}}$ is associated with the higher rate of both access and non-access site bleedings, but similar rates of MACE at 30 days compared to the StarClose SE $®$ device.
\end{abstract}

Key words: percutaneous coronary intervention, transfemoral access, transradial access, femoral closure, vascular closure devices

\section{Introduction}

Arterial access site bleeding is considered to be one of the most relevant complications associated with adverse clinical outcomes in patients undergoing percutaneous coronary intervention (PCI) [1]. A recent meta-analysis revealed a significantly increased risk of periprocedural mortality in patients undergoing PCI with concomitant access site bleeding (risk ratio [RR] 1.71, 95\% confidence interval [CI] 1.37 - 2.13) [2].

Recently, due to its advantage of reduced access site bleeding a transradial access (TRA) is regarded as the preferred approach for PCI [3, 4]. Campelo-Parada 
et al. demonstrated a significantly lower rate of long-term major adverse cardiac events (MACE) in patients undergoing PCI using TRA compared to those with transfemoral access (TFA) [5]. Nonetheless, TFA still remains the most commonly used approach because of several potential drawbacks of TRA, e.g., higher frequency of crossover to alternative vascular access, longer procedure time, inability to insert mechanical circulatory support devices, and risk for potential arterial conduits for bypass graft surgery [6].

To improve the efficiency of hemostasis, especially following femoral PCI vascular closure devices (VCD) were developed continuously over the last decades, although manual compression and sequential application of pressure bandages is often used [7]. Numerous prior trials demonstrated that the application of VCD being based on collagen plug, clip, or suture mechanisms might significantly decrease femoral access site bleeding in patients undergoing diagnostic cardiac catheterization as well as PCI compared to conventional manual compression $[8,9]$. Furthermore, Chodor et al. revealed no significant benefit of TRA in access site bleeding compared to TFA with consecutive use of StarClose SE® for femoral closure (FC) in patients with ST segment elevation myocardial infarction (STEMI) [10].

However, direct comparisons between various VCDs in terms of their efficacy in the interventional settings have been rarely investigated. Therefore, this study aims to compare directly one specific intravascular FC device (AngioSeal ${ }^{\mathrm{TM}}$, St. Jude Medical, Inc., St. Paul, MN, U.S.A.) with one specific extravascular FC device (StarClose SE®, Abbott, Illinois, U.S.A.) in patients after PCI focusing on overall and access site bleedings as well as MACE at short-term follow-up.

\section{Methods}

\section{Study population}

The present study was conducted as a single-center, prospective, nonrandomized study being performed at the First Department of Medicine, University Medical Centre Mannheim (UMM) in Mannheim, Germany. The study was designed as an open-label, observational all-comers study in order to recruit a consecutively generalizable and representative study population comparable to the daily practice in other PCI centers. The study was carried out according to the principles of the Declaration of Helsinki and was approved by the medical ethics commission II of the Medical Faculty Mannheim, University of Heidelberg, Germany. Written informed consent was obtained from all participating patients or their legal representatives.
Patients being planned for PCI were screened at our cardiologic department and included consecutively to this study, when they were subsequently treated either with intravascular closure device (AngioSeal ${ }^{\mathrm{TM}}$ ) or with extravascular closure device (StarClose SE®) after femoral PCI. Patients being treated with other VCD than AngioSeal ${ }^{\mathrm{TM}}$ or StarClose SE® after PCI were excluded. Patients with unsuccessful implantation of the AngioSeal ${ }^{\mathrm{TM}}$ or the StarClose SE® device immediately after PCI in the catherization laboratory were excluded. Further inclusion and exclusion criteria accorded to criteria of "The Femoral Closure versus Radial Compression Devices Related to Percutaneous Coronary Interventions" (FERARI, clinicaltrials.gov identifier: NCT02455661) study being outlined in detail in the previously published method paper [11]. According to an estimation of the power using the data of the first 100 patients, a sample size of 200 patients in each group was necessary to power the study sufficiently for the primary endpoint. Therefore, 200 consecutive patients were recruited in both groups [11].

\section{Procedure}

Conduction of PCI procedure (i.e., choice of access site, sheath diameter, used technique and PCI materials) was not influenced by the study protocol and based on the operator's discretion. Procedures with switching of access site were excluded. Heparin was used to achieve an activated clotting time (ACT) of 250-300 s during PCI and ACT was measured frequently. Peri-interventional additional antithrombotic treatment (i.e., bivalirudin or abciximab) as well as post-interventional loading with antiplatelet therapy were carried out according to European guidelines [12].

Femoral closure (FC) was achieved using the AngioSeal ${ }^{\mathrm{TM}}$ or the StarClose SE® device applied by experienced interventional cardiologists $(\geq 100$ applications each). Following the FC, a conventional pressure band was located in a standard fashion around the hips for $6 \mathrm{~h}$. Subsequently, these patients were checked for peripheral perfusion, motor function and sensibility regularly.

The Angio-Seal ${ }^{\mathrm{TM}}$ is composed of an absorbable polymer anchor compressing the inner vascular wall and an absorbable collagen sponge compressing the outer vascular wall. According to the instructions of use, an insertion sheath and an arteriotomy locator are snapped together and positioned with an introducer right in the femoral artery noticeable of the blood reflow. After removing the insertion sheath and introducer, the Angio-Seal ${ }^{\mathrm{TM}}$ device is inserted through the locator. A clicking sound indicates that the anchor has left the sheath. Pulling back the device, 
the anchor was pressed against the inner vessel wall and further retreat released the collagen plug in the exterior puncture hole. After removing the whole components of the device, a suture tube appeared. Applying pressure downward and meanwhile pulling back the device hub, the collagen compacts the outer vessel wall. A black mark is released, which should be cut close to the skin [11].

The StarClose SE® contains an introducer sheath, dilator, guidewire, and clip applier with a star shaped nitinol clip. When the primary procedure is completed, the catheter is removed and the sheath is left in place or exchanged for a StarClose SE® compatible sheath. The clip applier is attached to the introducer sheath, signaled by a loud click to the operator. A button on the device is depressed to expand the flexible wings in the artery and provide the user a tactile signal of being against the anterior femoral artery. The device is applied with light traction against the arteriotomy, then a "no tension" position while stabilizing the device is assumed. A sliding element on the body of the device is then advanced, splitting the sheath as the clip is advanced to the arteriotomy. The operator is signaled the completion of the sheath splitting by another loud click. While pressing down with the device, a trigger button is depressed to deploy the clip. Subsequently, the clip applier and introducer sheath are withdrawn. The nitinol clip provides a secure extravascular closure that does not invade the vessel lumen [13].

\section{Data acquisition}

Baseline characteristics, past medical history including chronic kidney (glomerular filtration rate $<60 \mathrm{ml} / \mathrm{min}$ ) or liver disease, heart failure (according to left ventricular ejection fraction) as well as laboratory values (i.e. creatinine, hemoglobin, platelet count and International Normalized Ratio (INR)) were collected from the in-hospital documentation system. All patients were followed up during hospital stay and until 30 days after the index procedure directly and by standardized telephone visits.

\section{Definition of study endpoints}

The primary endpoint was defined by the overall rate of all relevant access site and non-access site bleedings within 30 days following PCI. Overall bleedings were classified according to established criteria such as the "Bleeding Academic Research Consortium" (BARC), "The Thrombolysis in Myocardial Infarction" (TIMI), and "The Global Use of Strategies to Open Occluded Arteries" (GUSTO) [14-16]. Access site complications were defined as hematomas, active bleedings, dissections, pseudoaneurysms, arteriovenous fistulae, and retroperitoneal hematomas [17]. Access site bleedings were classified according to the FERARI classification [11].

The secondary endpoint consisted of MACE within 30 days of follow-up, which comprised all-cause and cardiovascular death, myocardial infarction, stent thrombosis, target lesion revascularization (TLR) as well as target vessel revascularization (TVR).

\section{Statistical analysis}

Statistical analysis was performed using SPSS Statistics (IBM, Armonk, NY) and GraphPad Prism (GraphPad Software, Inc., La Jolla, CA). Data are presented as medians with interquartile ranges $\left(25^{\text {th }}\right.$ to $75^{\text {th }}$ percentiles) or as total numbers with group-related percentages. The $p$-values $<0.05$ were considered statistically significant, $p$-values $<0.01$ were considered as a statistical trend. Normal distribution of data was tested with the Kolmogorov-Smirnov test. For data with normal distribution, the Student $t$ test was applied. Categorical variables were compared using the Chi-squared test; in case of low event rates the Fischer's exact test was applied. Baseline characteristics, which were shown to differ significantly between the two groups, were adjusted using uni- and multivariate logistic regression analyses for the predefined study endpoints.

\section{Results}

\section{Baseline characteristics}

A total of 400 consecutive patients after PCI were enrolled in the present study. 200 patients were treated with the intravascular device AngioSeal ${ }^{\mathrm{TM}}$ and another 200 patients were treated with the extravascular device StarClose SE® following PCI.

In a total of 16 patients of the StarClose SE® group the implantation of the device was unsuccessful bedside in the cardiac catheterization laboratory (12 patients: technical failure, device unable to fix at the outer vessel site; 4 patients: insufficient closure with relevant unstoppable arterial bleeding directly after release). These 16 patients received additional application of the FemoStop ${ }^{\mathrm{TM}}$ (Abbott, Illinois, U.S.A) to ensure final hemostasis and were excluded from final analysis.

Table 1 displays the baseline characteristics between the AngioSeal ${ }^{\mathrm{TM}}$ and the StarClose SE® group. The AngioSeal ${ }^{\mathrm{TM}}$ was significantly more often performed in patients with stable angina pectoris $(p=$ $0.0001)$ or with a positive viability testing $(p=0.0004)$, whereas the StarClose SE® was more often used in patients with STEMI $(p=0.0001)$ or in angiographic control examinations $(p=0.015)$. Patients in the 
AngioSeal $^{\mathrm{TM}}$ group suffered more often from peripheral vascular disease and underwent more often surgery of coronary artery bypass grafting (CABG). The rate of AngioSeal ${ }^{\mathrm{TM}}$ application was significantly higher in patients undergoing PCI with 5 F sheath diameter $(p=0.001)$, whereas the StarClose $\mathrm{SE} \AA$ was more often performed in patients undergoing PCI with $6 \mathrm{~F}$ sheath diameter $(p=0.009)$.
No significant differences of preexisting antiplatelet or anticoagulation therapy before PCI between both groups were observed except for acetylsalicylic acid (ASA) (73\% for StarClose SE® group and $56 \%$ for the AngioSeal ${ }^{\mathrm{TM}}$ group, $p=0.002$ ) and low molecular weight heparin $(\mathrm{LMWH})$ (1 patient in the StarClose $\mathrm{SE}{ }^{\circledR}$ group and 10 patients in the AngioSeal ${ }^{\mathrm{TM}}$ group, $p=0.011$ ) (Table 2).

Table 1. Baseline characteristics of $\mathrm{PCl}$ patients with application of vascular closure devices

\begin{tabular}{|c|c|c|c|c|}
\hline & All $(n=400)$ & StarClose $(n=200)$ & AngioSeal (n=200) & p value* \\
\hline Male, n (\%) & $286(71.5)$ & $151(75.5)$ & $135(67.5)$ & 0.077 \\
\hline Age, years (IQR) & $68(59-78)$ & $67(57-77)$ & $71(61-78)$ & 0.045 \\
\hline Height, cm (IQR) & $172(165-178)$ & $172(165-178)$ & $171(165-178)$ & 0.242 \\
\hline Weight, kg (IQR) & $81(70-91)$ & $81(70-90)$ & $81(72-92)$ & 0.377 \\
\hline BMI, kg/m² (IQR) & $27(24-30)$ & $27(24-30)$ & $28(24-31)$ & 0.128 \\
\hline \multicolumn{5}{|l|}{ Indication, $\mathbf{n}(\%)$} \\
\hline Stable AP & $55(13.8)$ & $5(2.5)$ & $50(25)$ & 0.0001 \\
\hline Unstable AP & $65(16.3)$ & $30(15)$ & 35 (17.5) & 0.498 \\
\hline NSTEMI & $107(26.8)$ & $60(30)$ & $47(23.5)$ & 0.142 \\
\hline STEMI & $59(14.8)$ & $46(23)$ & $13(6.5)$ & 0.0001 \\
\hline Pos. viability testing & $12(3)$ & $1(0.5)$ & $11(5.5)$ & 0.0004 \\
\hline Angio. Control & $75(18.8)$ & $47(23.5)$ & $28(14)$ & 0.015 \\
\hline Arrhythmia & $9(2.3)$ & $4(2)$ & $5(2.5)$ & 1.000 \\
\hline Syncope & $7(1.8)$ & $2(1)$ & $5(2.5)$ & 0.449 \\
\hline Heart failure & $9(2.3)$ & $3(1.5)$ & $6(3)$ & 0.503 \\
\hline Others & $2(0.5)$ & $2(1)$ & $0(0)$ & 0.499 \\
\hline \multicolumn{5}{|c|}{ Cardiovascular risk factors, $\mathrm{n}(\%)$} \\
\hline Arterial Hypertension & $302(75.5)$ & $147(73.5)$ & $155(77.5)$ & 0.352 \\
\hline Diabetes mellitus & $129(32.3)$ & $61(30.5)$ & $68(34)$ & 0.454 \\
\hline \multicolumn{5}{|l|}{ Smoking, each $n(\%)$} \\
\hline Active & $103(25.8)$ & $67(33.5)$ & $36(18)$ & 0.0004 \\
\hline Past & $66(16.5)$ & $28(14)$ & $38(19)$ & 0.178 \\
\hline Dyslipidemia & $162(40.5)$ & $77(38.5)$ & $85(42.5)$ & 0.415 \\
\hline Cardiac family history & $80(20)$ & $47(23.5)$ & $33(16.3)$ & 0.080 \\
\hline \multicolumn{5}{|l|}{ Prior medical history, $n(\%)$} \\
\hline Coronary artery disease & $196(49)$ & $95(47.5)$ & $101(50.5)$ & 0.548 \\
\hline CABG & $31(7.8)$ & $9(4.5)$ & $22(11)$ & 0.015 \\
\hline Peripher vascular disease & $21(5.3)$ & $6(3)$ & $15(7.5)$ & 0.044 \\
\hline Stroke/TIA & $24(6)$ & $10(5)$ & $14(7)$ & 0.400 \\
\hline Heart valve surgery & $6(1.5)$ & $2(1)$ & $4(2)$ & 0.685 \\
\hline \multicolumn{5}{|l|}{ Atrial fibrillation, each } \\
\hline Paroxymal & $27(6.8)$ & $17(8.5)$ & $10(5)$ & 0.163 \\
\hline Persistent & $5(1.3)$ & $3(1.5)$ & $2(1)$ & 1.000 \\
\hline Permanent & $11(2.8)$ & $6(3)$ & $5(2.5)$ & 1.000 \\
\hline Non classified & $9(2.3)$ & $0(0)$ & $9(4.5)$ & 0.004 \\
\hline Pacemaker & $15(3.8)$ & $7(3.5)$ & $8(4)$ & 0.792 \\
\hline Implantable defibrillator & $13(3.3)$ & $6(3)$ & $7(3.5)$ & 0.778 \\
\hline Impaired liver function & $1(0.3)$ & $0(0)$ & $1(0.5)$ & 1.000 \\
\hline Prior GI bleeding & $8(2)$ & $5(2.5)$ & $3(1.5)$ & 0.475 \\
\hline LVEF, \% (median, IQR) & $45(39-55)$ & $50(44-57)$ & $44(30-50)$ & 0.001 \\
\hline \multicolumn{5}{|c|}{ Baseline laboratory values (median, IQR) } \\
\hline $\mathrm{Hb}, \mathrm{g} / \mathrm{dl}$ & $13.8(12.6-14.8)$ & $14.0(13.0-14.9)$ & $13.7(12.1-14.7)$ & 0.038 \\
\hline Serum creatinine, $\mathrm{mg} / \mathrm{dl}$ & $1.01(0.82-1.20)$ & $0.98(0.81-1.15)$ & $1.05(0.84-1.25)$ & 0.026 \\
\hline Thrombocytes, $10^{9} / 1$ & $220(182-263)$ & $222(187-266)$ & $212(179-261)$ & 0.296 \\
\hline INR & $1.01(0.97-1.06)$ & $1.01(0.98-1.07)$ & $1.01(0.96-1.06)$ & 0.175 \\
\hline \multicolumn{5}{|l|}{ Sheath diameter, $\mathbf{n}(\%)$} \\
\hline 5 French & $39(9.8)$ & $10(5)$ & $29(14.5)$ & 0.001 \\
\hline 6 French & $358(89.5)$ & $187(93.5)$ & $171(85.5)$ & 0.009 \\
\hline 7 French & $3(0.8)$ & $3(1.5)$ & $(0)$ & 0.248 \\
\hline Hospital stay, days (IQR) & $7(3-10)$ & $7(4-9)$ & $7(2-11)$ & 0.498 \\
\hline
\end{tabular}

$B M I$ body mass index, CABG coronary artery bypass grafting, $G$ I gastrointestinal, $H b$ hemoglobin, INR International Normalized Ratio, $L V E F$

left ventricular ejection fraction, (N)STEMI (non) ST-segment elevation myocardial infarction, TIA transient ischaemic attack.

* $p$ values for the comparison of femoral closure by StarClose versus femoral closure by AngioSeal group, significant $p$ values are in bold type $(p<0.05)$ 
Table 2. Antithrombotic therapies being used in the study

\begin{tabular}{|c|c|c|c|c|}
\hline & All $(n=400)$ & StarClose $(n=200)$ & AngioSeal (n=200) & p value* \\
\hline \multicolumn{5}{|c|}{ Prior antithrombotic treatment, $n(\%)$} \\
\hline ASA & $263(65.8)$ & $146(73)$ & $117(58.5)$ & 0.002 \\
\hline Clopidogrel & 66 (16.6) & $31(15.5)$ & $35(17.7)$ & 0.590 \\
\hline Prasugrel & $12(3)$ & $3(1.5)$ & $9(4.5)$ & 0.139 \\
\hline Ticagrelor & $7(1.8)$ & $5(2.5)$ & $2(1)$ & 0.449 \\
\hline \multicolumn{5}{|l|}{ Prior oral anticoagulation, $n(\%)$} \\
\hline Phenprocoumon & $29(7.3)$ & $15(7.5)$ & $14(7)$ & 0.847 \\
\hline Rivaroxaban & $8(2)$ & $3(1.5)$ & $5(2.5)$ & 0.724 \\
\hline Dabigatran & $6(1.5)$ & $3(1.5)$ & $3(1.5)$ & 1.000 \\
\hline Apixaban & $4(1)$ & $1(0.5)$ & $3(1.5)$ & 0.623 \\
\hline LMWH & $11(2.8)$ & $1(0.5)$ & $10(5)$ & 0.011 \\
\hline \multicolumn{5}{|c|}{ Antithrombotic Loading therapy during PCI, $n(\%)$} \\
\hline ASA & $7(1.8)$ & $0(0)$ & $7(3.5)$ & 0.015 \\
\hline Clopidogrel & $203(50.7)$ & $83(41.5)$ & $120(60)$ & 0.0002 \\
\hline Prasugrel & $41(10.3)$ & $27(13.5)$ & $14(7)$ & 0.032 \\
\hline Ticagrelor & $52(13)$ & $50(25)$ & $2(1)$ & 0.0001 \\
\hline ASA + clopidogrel & $46(11.5)$ & $21(10.5)$ & $25(12.5)$ & 0.531 \\
\hline ASA + prasugrel & $9(2.3)$ & $4(2)$ & $5(2.5)$ & 1.000 \\
\hline ASA + ticagrelor & $10(2.5)$ & $8(4)$ & $2(1)$ & 0.105 \\
\hline Bivalirudin application, $\mathrm{n}(\%)$ & $1(0.3)$ & $0(0)$ & $1(0.5)$ & 1.000 \\
\hline Abciximab application, $\mathrm{n}(\%)$ & $16(4)$ & $10(5)$ & $6(3)$ & 0.307 \\
\hline
\end{tabular}

ASA acetylsalicylic acid, $L M W H$ low molecular weight heparin

\section{Primary endpoint: bleeding complications within $\mathbf{3 0}$ days following $\mathbf{P C l}$}

As shown in Table 3 bleedings are classified according to BARC, TIMI, and GUSTO as well as FERARI. Due to bleeding events consisting mainly of minor hematomas, BARC type 1 bleeding constituted the majority of bleeding complications. BARC type 4 bleeding was not present in our study cohort because it is directly linked to CABG. For a similar reason, "minimal" in TIMI classification applied for $84 \%$ of bleeding events and only "mild" subgroup of GUSTO classification was existent in the StarClose SE® group. The rates of overall and non-access site bleeding were significantly higher in the AngioSeal ${ }^{\mathrm{TM}}$ group $(p=$ $0.012 ; p=0.003$ ), whereas access site bleedings did not significantly differ between both groups $(p>0.05)$ (Table 4). The significantly higher rate of non-access site bleeding in the AngioSeal ${ }^{\mathrm{TM}}$ group was shown to be related with increased bleeding requiring medical attention in TIMI classification, BARC Type 2 bleeding and mild GUSTO bleedings in this group ( $p$ $=0.008 ; p=0.0002 ; p=0.028$ ). Focusing on FERARI bleedings, a significantly higher rate of complicated bleeding including active bleeding, dissection, fistula, pseudoaneurysm, retroperitoneal hematoma, arterial occlusion, or need of surgical repair was observed in the AngioSeal ${ }^{\mathrm{TM}}$ group $(p=0.011)$.

\section{Secondary endpoint: MACE within 30 days following PCI}

In this study MACE occurred rarely and did not differ significantly between both groups (Table 4). None of the two deaths, which occurred within 30 days of follow-up, was related to any bleeding complication. In addition, no significant differences of TVR and TLR were observed in both groups.

Table 3. Comparison of bleedings according to bleeding classification systems in the study

\begin{tabular}{|c|c|c|c|c|}
\hline & $\begin{array}{l}\text { All } \\
(n=400)\end{array}$ & $\begin{array}{l}\text { StarClose } \\
(\mathrm{n}=200)\end{array}$ & $\begin{array}{l}\text { Angio Seal } \\
(n=200)\end{array}$ & p value ${ }^{*}$ \\
\hline \multicolumn{5}{|l|}{ BARC, n (\%) } \\
\hline Type 1 & $172(43.1)$ & $84(42)$ & $88(44)$ & 0.686 \\
\hline Type 2 & $21(5.3)$ & $2(1)$ & $19(9.5)$ & 0.0002 \\
\hline Type 3 & $6(1.5)$ & $1(0.5)$ & $5(2.5)$ & 0.215 \\
\hline Type 4 & $0(0)$ & $0(0)$ & $0(0)$ & 1.000 \\
\hline Type 5 & $0(0)$ & $0(0)$ & $0(0)$ & 1.000 \\
\hline \multicolumn{5}{|l|}{ TIMI, n (\%) } \\
\hline Minimal & $168(42)$ & 79 (39.5) & $89(44.5)$ & 0.311 \\
\hline $\begin{array}{l}\text { Requiring medical } \\
\text { attention }\end{array}$ & $30(7.5)$ & $8(4)$ & $22(11)$ & 0.008 \\
\hline Minor & $1(0.3)$ & $0(0)$ & $1(0.5)$ & 1.000 \\
\hline Major & $0(0)$ & $0(0)$ & $0(0)$ & 1.000 \\
\hline \multicolumn{5}{|l|}{ GUSTO, n (\%) } \\
\hline Mild & $196(49)$ & $87(43.5)$ & $109(54.5)$ & 0.028 \\
\hline Moderate & $1(0.3)$ & $0(0)$ & $1(0.5)$ & 1.000 \\
\hline $\begin{array}{l}\text { Severe or life } \\
\text { threatening }\end{array}$ & $2(0.5)$ & $0(0)$ & $2(1)$ & 0.499 \\
\hline \multicolumn{5}{|l|}{ FERARI, n (\%) } \\
\hline Small, $<5 \mathrm{~cm}$ & $93(23.3)$ & $51(25.5)$ & $42(21)$ & 0.287 \\
\hline Intermediate, $5-15 \mathrm{~cm}$ & $49(12.3)$ & $19(9.5)$ & $30(15)$ & 0.093 \\
\hline Large, $>15 \mathrm{~cm}$ & $33(8.3)$ & $15(7.5)$ & $18(9)$ & 0.586 \\
\hline Complicated ${ }^{1}$ & $11(2.8)$ & $1(0.5)$ & $10(5)$ & 0.011 \\
\hline
\end{tabular}

\section{Multivariable logistic regression analyses for the primary endpoint}

The primary endpoint was adjusted within multivariable logistic regression analyses including the following statistically different variables (Table 1 
and 2): Preexisting antiplatelet therapy, anticoagulation treatment before PCI with ASA or LMWH, mono loading following PCI with ASA, clopidogrel, prasugrel or ticagrelor, age, sheath size, peripheral vascular disease and renal function.

None of the above described variables were associated with consistent impact on the primary endpoint in multivariate logistic regression models (Table S1 and S2). Notably, neither sheath diameters nor antithrombotic therapies effected any bleeding.

Table 4. Primary and secondary endpoints in the study

\begin{tabular}{|c|c|c|c|c|}
\hline & $\begin{array}{l}\text { All } \\
(n=400)\end{array}$ & $\begin{array}{l}\text { StarClose } \\
(\mathbf{n}=200)\end{array}$ & $\begin{array}{l}\text { AngioSeal } \\
(n=200)\end{array}$ & $\begin{array}{l}\mathrm{p} \\
\text { value* }\end{array}$ \\
\hline \multicolumn{5}{|l|}{ Primary Endpoint } \\
\hline $\begin{array}{l}\text { Overall Bleedings (Access and } \\
\text { Non Access Site), } \mathrm{n}(\%)\end{array}$ & $199(49.8)$ & $87(43.5)$ & $112(56)$ & 0.012 \\
\hline Non Access Site Bleedings, n (\%) & $13(3.3)$ & $1(0.5)$ & $12(6)$ & 0.003 \\
\hline \multicolumn{5}{|l|}{ Access Site Bleedings, each n (\%) } \\
\hline Hematoma & $175(43.8)$ & $85(42.5)$ & $90(45)$ & 0.614 \\
\hline Bleeding & $2(0.5)$ & $0(0)$ & $2(1)$ & 0.499 \\
\hline Dissection & $3(0.8)$ & $1(0.5)$ & $2(1)$ & 1.000 \\
\hline Fistula & $1(0.3)$ & $0(0)$ & $1(0.5)$ & 1.000 \\
\hline Aneurysm & $5(1.3)$ & $0(0)$ & $5(2.5)$ & 0.061 \\
\hline $\begin{array}{l}\text { Re-hospitalization due to access } \\
\text { site bleeding, } \mathrm{n}(\%)\end{array}$ & $2(0.5)$ & $0(0)$ & $2(1)$ & 0.499 \\
\hline \multicolumn{5}{|l|}{ MACE } \\
\hline Death within follow-up, n (\%) & $2(0.5)$ & $1(0.5)$ & $1(0.5)$ & 1.000 \\
\hline Myocardial infarction, n (\%) & $2(0.5)$ & $0(0)$ & $2(1)$ & 0.499 \\
\hline Stent thrombosis, $\mathrm{n}(\%)$ & $2(0.5)$ & $1(0.5)$ & $1(0.5)$ & 1.000 \\
\hline TLR, n (\%) & $3(0.8)$ & $2(1)$ & $1(0.5)$ & 1.000 \\
\hline TVR, n (\%) & $2(0.5)$ & $2(1)$ & $0(0)$ & 0.499 \\
\hline Stroke, n (\%) & $2(0.5)$ & $0(0)$ & $2(1)$ & 0.499 \\
\hline
\end{tabular}

\section{Discussion}

The present study compared directly two mechanistically different types of FC devices (AngioSeal ${ }^{\mathrm{TM}}$ versus StarClose SE®) were focusing on bleedings and MACE in patients undergoing PCI. In case of successful implantation, FC by the extravascular StarClose SE® was significantly associated with lower rates of overall and non-access bleeding as well as complicated access site bleeding compared to the intravascular AngioSeal ${ }^{\mathrm{TM}}$. However, the other types of access site bleeding including small, intermediate, or large hematomas did not differ significantly between both groups. None of the above described univariable significant risk factors had consistent impact on the primary endpoint after multivariate adjustment.

Recent studies suggested that both arterial access site and non-access site bleedings following PCI were significantly associated with increased short- as well as long-term mortality regardless of bleedings origin. $[15,18]$. Accordingly, multidisciplinary approaches with improved medical therapy and innovative interventional closure devices as well as techniques have been developed to minimize risk of bleeding and to improve consequently the clinical outcomes [19, 20]. Especially, in the case of access site bleeding, TRA was shown to decrease significantly the rate of procedure related bleedings as well as short- and long-term mortality compared to TFA in many recent prior studies [21-23]. Notwithstanding, TFA is still frequently utilized because of the above mentioned disadvantages of TRA [24], especially in patients with high-risk STEMI and complex PCI [25].

In order to compensate higher risk of bleeding in using TFA compared to TRA, the application of VCDs was already proposed in the early 1990's. [26, 27]. In a clinical trial by Gregory et al. the risk of vascular complications was significantly lower with VCD (AngioSeal ${ }^{\mathrm{TM}}$ ) compared to manual compression both in patients undergoing diagnostic coronary angiography (OR 0.43, 95\% CI 0.31 - 0.60) and PCI (OR $0.51,95 \% 0.31$ - 0.81) [28]. Furthermore, Sanborn et al. demonstrated decreased adverse clinical outcomes in STEMI patients undergoing PCI with application of VCD compared to manual compression (hazard ratio [HR] 0.61, 95\% CI $0.42-0.89, p=0.009$ ) [29].

However, it is still debatable which kind of VCDs may be the preferred device in terms of efficacy and safety. Schulz-Schupke et al. demonstrated in their randomized study that vascular access site complications were not statistically but numerically lower in patients assigned to the intravascular VCD compared to the extravascular VCD after diagnostic coronary angiography [8]. Additionally, both time to adequate hemostasis and closure device failures were significantly lower in patients with application of the intravascular VCD. These more favorable results of hemostasis with the intravascular compared to the extravascular VCD may be explained by the tighter fixation resulting from more tension of an intravascular VCD. Contrastively, in another study investigated in a diagnostic setting by Veasey et al. the extravascular VCD (StarClose SE®) was significantly associated with less hematoma one week post-procedure compared to the intravascular VCD (AngioSeal ${ }^{\mathrm{TM}}$ ) [30].

Following PCI with concomitant anticoagulation, the application of AngioSeal ${ }^{\mathrm{TM}}$ was shown to reduce significantly the rate of TIMI minor (5.5\% for AngioSeal ${ }^{\mathrm{TM}}, 6.9 \%$ for StarClose SE®, $p<$ 0.01), TIMI major (1.2\% for AngioSeal ${ }^{\mathrm{TM}}, 2.4 \%$ for StarClose SE®, $p<0.05)$, and all bleeding complications $\left(9.2 \%\right.$ for AngioSeal ${ }^{\mathrm{TM}}, 10.2 \%$ for StarClose SE®, $p<0.001)$ compared to StarClose SE® irrespective of anticoagulation [31]. However, the intravascular VCD was significantly associated with a 
higher rate of surgical repair due to distal embolization of either the anchor footplate alone or in combination with thrombus compared to extravascular VCD $\left(0.7 \%\right.$ for AngioSeal ${ }^{\mathrm{TM}}, 0.2 \%$ for StarClose SE®, $p<0.05)$. Yeni et al., however, found no significant differences of vascular and bleeding complications between intravascular and extravascular devices after PCI [32]. Interestingly, a recent comparison of AngioSeal ${ }^{\mathrm{TM}}$ and StarClose SE® in non-cardiological procedures revealed also no statistically significant difference between both groups in terms of bleedings and time to hemostasis [33].

Remarkably, in contrast to other prior studies, the present compared directly two mechanistically different VCDs after PCI and revealed that the intravascular AngioSeal ${ }^{\mathrm{TM}}$ was associated with significantly rate of overall, non-access as well as complicated access site bleeding compared to extravascular StarClose SE®.

Despite the use of both VCDs the rates of access site bleeding appeared to be higher than expected in the FERARI study. Access site bleedings were shown in about $43 \%$ of patients in the StarClose SE® group and $50 \%$ of patients in the AngioSeal ${ }^{\mathrm{TM}}$ group. The higher rate of procedure related bleedings might be explained by the more detailed discrimination of minor bleedings within FERARI classification. In contrast to other bleeding classifications the FERARI classification reflect more precisely most common types of exercised bleeding and hematomas smaller than $5 \mathrm{~cm} \quad(23.3 \%)$. These small hematomas contributed to low-graded bleedings within the other classification systems, i.e. BARC type 1, TIMI minimal, and GUSTO mild.

Many previous studies assessed a significant association of major bleedings following PCI with major adverse outcomes [34, 35]. However, in the present study the difference of bleeding rates in both treatment groups did not affect the development of MACE. Furthermore, no significant differences of TLR or TVR rates were observed in between AngioSeal $^{\mathrm{TM}}$ and StarClose SE® group.

\section{Conclusions}

In case of successful implantation, FC by the AngioSeal ${ }^{\mathrm{TM}}$ is associated with higher rates of both access and non-access site bleedings. However, no significant difference of MACE at 30 days was observed in the AngioSeal $^{\mathrm{TM}}$ and StarClose SE® group. The FERARI classification was shown to better discriminate access site complications following PCI.

\section{Supplementary Material}

Supplementary tables.

http://www.medsci.org/v16p0043s1.pdf

\section{Acknowledgments}

This study was supported by the Deutsches Zentrum fur Herz-Kreislauf-Forschung - German Centre for Cardiovascular Research (DZHK).

\section{Competing Interests}

The authors have declared that no competing interest exists.

\section{References}

1. Byrne RA, Cassese S, Linhardt M, et al. Vascular access and closure in coronary angiography and percutaneous intervention. Nat Rev Cardiol. 2013; 10:27-40.

2. Kwok CS, Khan MA, Rao SV, et al. Access and non-access site bleeding after percutaneous coronary intervention and risk of subsequent mortality and major adverse cardiovascular events: systematic review and meta-analysis. Circ Cardiovasc Interv. 2015; 8 .

3. Ratib K, Mamas MA, Anderson SG, et al. Access site practice and procedural outcomes in relation to clinical presentation in 439,947 patients undergoing percutaneous coronary intervention in the United kingdom. JACC Cardiovasc Interv. 2015; 8:20-9.

4. Joyal D, Bertrand OF, Rinfret S, et al. Meta-analysis of ten trials on the effectiveness of the radial versus the femoral approach in primary percutaneous coronary intervention. Am J Cardiol. 2012; 109:813-8.

5. Campelo-Parada F, Carrie D, Bartorelli AL, et al. Radial Versus Femoral Approach for Percutaneous Coronary Intervention: MACE Outcomes at Long-Term Follow-up. J Invasive Cardiol. 2018; 30:262-8.

6. Anderson HV, Faxon DP. Balanced Adoption of Radial Artery Access for Primary Percutaneous Coronary Intervention. JAMA Cardiol. 2017; 2:1059-60.

7. Dauerman HL, Applegate RJ, Cohen DJ. Vascular closure devices: the second decade. J Am Coll Cardiol. 2007; 50:1617-26.

8. Schulz-Schupke S, Helde S, Gewalt S, et al. Comparison of vascular closure devices vs manual compression after femoral artery puncture: the ISAR-CLOSURE randomized clinical trial. JAMA. 2014; 312:1981-7.

9. Tavris DR, Wang Y, Jacobs S, et al. Bleeding and vascular complications at the femoral access site following percutaneous coronary intervention (PCI): an evaluation of hemostasis strategies. J Invasive Cardiol. 2012; 24:328-34.

10. Chodor P, Kurek T, Kowalczuk A, et al. Radial vs femoral approach with StarClose clip placement for primary percutaneous coronary intervention in patients with ST-elevation myocardial infarction. RADIAMI II: a prospective, randomised, single centre trial. Kardiol Pol. 2011; 69:763-71.

11. Behnes M, Unsal M, Hoffmann U, et al. Design and Rationale of the Femoral Closure versus Radial Compression Devices Related to Percutaneous Coronary Interventions (FERARI) Study. Clin Med Insights Cardiol. 2015; 9:97-103.

12. Authors/Task Force m, Windecker S, Kolh P, et al. 2014 ESC/EACTS Guidelines on myocardial revascularization: The Task Force on Myocardial Revascularization of the European Society of Cardiology (ESC) and the European Association for Cardio-Thoracic Surgery (EACTS)Developed with the special contribution of the European Association of Percutaneous Cardiovascular Interventions (EAPCI). Eur Heart J. 2014; 35:2541-619.

13. Hermiller JB, Simonton C, Hinohara T, et al. The StarClose Vascular Closure System: interventional results from the CLIP study. Catheter Cardiovasc Interv . 2006; 68:677-83.

14. Mehran R, Rao SV, Bhatt DL, et al. Standardized bleeding definitions for cardiovascular clinical trials: a consensus report from the Bleeding Academic Research Consortium. Circulation. 2011; 123:2736-47.

15. Vavalle JP, Clare R, Chiswell K, et al. Prognostic significance of bleeding location and severity among patients with acute coronary syndromes. JACC Cardiovasc Interv. 2013; 6:709-17.

16. Bovill EG, Terrin ML, Stump DC, et al. Hemorrhagic events during therapy with recombinant tissue-type plasminogen activator, heparin, and aspirin for acute myocardial infarction. Results of the Thrombolysis in Myocardial Infarction (TIMI), Phase II Trial. Ann Intern Med. 1991; 115:256-65.

17. Stone PA, Campbell JE. Complications related to femoral artery access for transcatheter procedures. Vasc Endovascular Surg. 2012; 46:617-23.

18. Rao SV, O'Grady K, Pieper KS, et al. Impact of bleeding severity on clinical outcomes among patients with acute coronary syndromes. Am J Cardiol. 2005; 96:1200-6

19. Nairooz R, Sardar P, Amin H, et al. Meta-analysis of randomized clinical trials comparing bivalirudin versus heparin plus glycoprotein IIb/IIIa inhibitors in 
patients undergoing percutaneous coronary intervention and in patients with ST-segment elevation myocardial infarction. Am J Cardiol. 2014; 114:250-9.

20. Ndrepepa G, Neumann FJ, Deliargyris EN, et al. Bivalirudin versus heparin plus a glycoprotein $\mathrm{IIb} / \mathrm{IIIa}$ inhibitor in patients with non-ST-segment elevation myocardial infarction undergoing percutaneous coronary intervention after clopidogrel pretreatment: pooled analysis from the ACUITY and ISAR-REACT 4 trials. Circ Cardiovasc Interv. 2012; 5:705-12.

21. Koifman E, Gaglia MA, Jr., Escarcega RO, et al. Comparison of transradial and transfemoral access in patients undergoing percutaneous coronary intervention for complex coronary lesions. Catheter Cardiovasc Interv. 2017; 89:640-6.

22. Dery JP, Mahaffey KW, Tricoci P, et al. Arterial access site and outcomes in patients undergoing percutaneous coronary intervention with and without vorapaxar. Catheter Cardiovasc Interv. 2016; 88:163-73.

23. Porto I, Bolognese L, Dudek D, et al. Impact of Access Site on Bleeding and Ischemic Events in Patients With Non-ST-Segment Elevation Myocardial Infarction Treated With Prasugrel: The ACCOAST Access Substudy. JACC Cardiovasc Interv. 2016; 9:897-907.

24. Basu D, Singh PM, Tiwari A, et al. Meta-analysis comparing radial versus femoral approach in patients 75 years and older undergoing percutaneous coronary procedures. Indian Heart J. 2017; 69:580-8.

25. Hillegass WB, Brott BC. Can femoral access bleeding outcomes be improved? Catheter Cardiovasc Interv. 2015; 85:380-1.

26. Cox T, Blair L, Huntington C, et al. Systematic Review of Randomized Controlled Trials Comparing Manual Compression to Vascular Closure Devices for Diagnostic and Therapeutic Arterial Procedures. Surg Technol Int. $2015 ; 27: 32-44$.

27. Tavris DR, Gallauresi BA, Lin B, et al. Risk of local adverse events following cardiac catheterization by hemostasis device use and gender. J Invasive Cardiol. 2004; 16:459-64.

28. Gregory D, Midodzi W, Pearce N. Complications with Angio-Seal vascular closure devices compared with manual compression after diagnostic cardiac catheterization and percutaneous coronary intervention. J Interv Cardiol. 2013; 26:630-8.

29. Sanborn TA, Tomey MI, Mehran R, et al. Femoral vascular closure device use, bivalirudin anticoagulation, and bleeding after primary angioplasty for STEMI: results from the HORIZONS-AMI trial. Catheter Cardiovasc Interv. 2015; 85:371-9.

30. Veasey RA, Large JK, Silberbauer J, et al. A randomised controlled trial comparing StarClose and AngioSeal vascular closure devices in a district general hospital--the SCOAST study. Int J Clin Pract. 2008; 62:912-8.

31. Iqtidar AF, Li D, Mather J, et al. Propensity matched analysis of bleeding and vascular complications associated with vascular closure devices vs standard manual compression following percutaneous coronary intervention. Conn Med. 2011; 75:5-10.

32. Yeni H, Axel M, Ornek A, et al. Clinical and Subclinical Femoral Vascular Complications after Deployment of two Different Vascular Closure Devices or Manual Compression in the Setting of Coronary Intervention. Int J Med Sci. 2016; 13:255-9.

33. Lucatelli P, Fanelli F, Cannavale A, et al. Angioseal VIP(R) vs. StarClose SE(R) closure devices: a comparative analysis in non-cardiological procedures. J Cardiovasc Surg (Torino). 2017; 58:80-6.

34. Verheugt FW, Steinhubl SR, Hamon M, et al. Incidence, prognostic impact, and influence of antithrombotic therapy on access and nonaccess site bleeding in percutaneous coronary intervention. JACC Cardiovasc Interv. 2011; 4:191-7.

35. Moscucci M, Fox KA, Cannon CP, et al. Predictors of major bleeding in acute coronary syndromes: the Global Registry of Acute Coronary Events (GRACE). Eur Heart J. 2003; 24:1815-23. 